\title{
Characteristics of A-waves in patients with amyotrophic lateral sclerosis (ALS) versus demyelinating neuropathies (DN)
}

\author{
Hebatallah R Rashed
}

\begin{abstract}
Objective: Detect the occurrence and characteristics of A-waves in amyotrophic lateral sclerosis (ALS) and demyelinating neuropathies (DN).

Methodology: Nerve conduction studies (NCS) of 28 patients (14 patients with ALS and 14 patients with DN) were reanalyzed to look for A-waves.

Results: We studied 61 nerves of the ALS group and 64 nerves of DN. In the ALS group, A-waves were detected in 21 nerves out of 61 nerves (34.4\%). In the DN group, A-waves were recorded from 25 nerves out of 64 nerves (39\%). In the DN group, A-waves were detected more often in nerves of upper extremities than lower extremities (out of a total of 25 nerves with A-waves, 18 median and ulnar nerves showed A-waves (72\%) and that was statistically significant $(P=0.0021)$ ). Complex A-waves (CAWs) occurred exclusively in patients with DN.
\end{abstract}

Conclusion: Most of the A-waves in the DN group were recorded more frequently from nerves of the upper extremities than those from lower extremities. CAWs occurred only in DN.

Significance: CAW seems to be a useful specific electrodiagnostic tool to distinguish DN from ALS.

Keywords: Demyelinating neuropathies, NCS, ALS, A-waves

\section{Introduction}

A-waves are late motor responses that have the same latencies, amplitudes, and morphology with every electrical stimulus [9]. The exact pathophysiologic mechanism responsible for the occurrence of A-waves remains unclear. There are several mechanisms that could be responsible for the generation of A-waves, including proximal reexcitation or ephaptic transmission [9].

A-waves were considered a nonspecific finding of unclear electrodiagnostic and clinical significance. However, several studies have found that A-waves could be an indicator of peripheral nerve dysfunction. A-waves are commonly seen in acute inflammatory demyelinating polyradiculoneuropathy (AIDP) [6]. Some studies have shown that they may be considered as a promising novel marker for demyelination. In their study, Rowin and Meriggioli [8] found that Awaves are prevalent in hereditary and acquired neuropathies and they often have complex morphology. However, they found that A-waves recorded from tibial nerves are not considered to be abnormal when found in an otherwise normal study [8].

Some studies reported that A-wave could be found also in motor neuron disease patients, and its presence is indicative of lower motor neuron dysfunction $[4,8]$. In their study, Fang and her colleagues [4] found that the frequency of occurrence of A-waves is significantly higher in patients with amyotrophic lateral sclerosis (ALS) compared to normal subjects [4]. However, studies about the clinical significance of A-waves in ALS remain scarce.

The differentiation between demyelinating neuropathies (DN) and ALS is important for prognosis and therapy. However, that can be difficult on the clinical bases especially when sensory signs and symptoms are missing in demyelinating neuropathies, as multifocal motor neuropathy (MMN) for example [10]. Thus, finding new tools for separating both conditions is crucial.

Correspondence: Rashedheba83@gmail.com

Ain Shams University Hospital, 12 Abadir St, Alzaytoun, Cairo, Egypt 
The aim of the current study is to detect the occurrence of A-waves in ALS and DN and determine the characteristics of A-waves in both conditions.

\section{Patients and methodology}

Nerve conduction studies (NCS) of 28 patients (14 patients with motor neuron disease and 14 patients with demyelinating neuropathy) referred to the Department of Neurology of Ain Shams University hospital were retrieved for reanalysis to look for A-waves. This is a retrospective study that was approved by the local ethical committee of Neurology Department, Ain Shams University. Consent was obtained from all patients. The electrophysiological tests did not exceed the usual procedure performed in our laboratory and no extra tests were done to the patients, and we did not include any personal data in our results. Studies were done in the period between December 2018 and May 2019.

Inclusion criteria:

1) Male or female

2) Age $\geq 18$

3) Patients with demyelinating neuropathy must have definite/probable/possible criteria for demyelination in electrodiagnostic studies [3].

4) Patients with ALS must have definite/probable/ possible criteria for ALS according to the revised El Escorial criteria [2].

5) Patients in both groups were newly diagnosed with DN/ALS and were not on specific treatment at the time of performing the study.

\section{Exclusion criteria:}

1) Known risk factor for peripheral neuropathy (e.g., diabetes mellitus, renal failure, and chronic alcohol abuse)

Criteria for the identification of A-waves were $[1,5]$ (1) stable amplitude and configuration, (2) variation of onset latencies by less than $1.5 \mathrm{~ms}$, and (3) present in at least $40 \%$ of the recorded responses. Complex A-waves (CAWs) are defined as greater than three phases, desynchronization, or $>2$ discreet biphasic potentials [7].

For the definition of demyelinating polyneuropathy, we used the criteria proposed by EFNS Task Force/CME Article [3]. All recordings met the criteria for definite/ probable/possible demyelination.

Recordings were performed on Nicolet Viking quest EMG, NCS, and EP, two channel (Natus, USA) devices and responses recorded using surface electrodes. Motor nerve conduction was investigated in the median (recording from the abductor pollicis muscle after stimulation at the wrist, elbow, axilla, and Erb's point), ulnar (recording from abductor digiti minimi muscle after stimulation up at the wrist, below elbow, above elbow, axilla, and Erb's point), tibial (recording from muscle abductor hallucis after stimulation behind medial malleous and at popliteal fossa), and peroneal nerves (recording from extensor digitorum brevis muscle after stimulation at the ankle, below and above fibular head). During the examination, the skin temperature at the ankle and wrist was kept above $32^{\circ} \mathrm{C}$. We used standard silver/silver chloride surface electrodes ( $10 \mathrm{~mm}$ in diameter), with the active electrode placed on the belly of the muscle and the reference electrode positioned on a muscle tendon. Electrical stimulation was performed at the distal stimulation site using repetitive stimulation at a frequency of $0.5 \mathrm{~Hz}$, the sweep speed should be increased to 5 or $10 \mathrm{~ms}$, and the gain is $200 \mu \mathrm{V}$ and a duration of $0.2 \mathrm{~ms}$ (for each stimuli). A submaximal stimulation is needed to elicit Awaves, followed by supramaximal stimulation to elicit Fwaves. Amplitudes were measured peak-to-peak (> $50 \mu \mathrm{V})$. To differentiate A-waves from late components of dispersed $M$ responses, the stimulation was repeated at few centimeters proximally. Late responses were recorded in each motor nerve when stimulated at the wrist (median or ulnar) or the ankle (peroneal or tibial).

\section{Statistical methods}

The results are expressed as mean \pm standard deviation. Statistical significance was calculated using chi-squared test and Fisher's exact test for comparison of categorical data and two-sample $t$ test to determine the significant difference between means. For all statistical analysis tests, a probability of $P<0.05$ was considered statistically significant.

\section{Results}

This is a retrospective study in which NCS of 14 patients with ALS (a total of 61 nerves were tested) and 14 patients with $\mathrm{DN}$ (a total of 64 nerves were tested) were reanalyzed. All the 14 patients with ALS, 10 males and 4 females with mean age $55.35 \pm 11.3$ years (range $38-73$ ), were diagnosed with definite, probable, or possible ALS according to the revised El Escorial criteria. All the 14 patients with DN, 8 males and 6 females with mean age $60.64 \pm 13.1$ years (range 34-75), were diagnosed with definite, probable, or possible demyelinating neuropathy. There was no statistically significant age difference between both groups $(P=0.263)$. The male to female ratio in both groups did not show a significant departure from the 1:1 ratio; the Fisher exact test statistic value is 0.6946 which is not statistically significant.

Patients in both groups were newly diagnosed with DN/ALS and were not on specific treatment at the time of the study. The duration of illness was $\leq 1$ year among patients in the DN group and $\leq 2$ years among patients in the ALS group. 
The frequency of occurrence of A-waves was slightly higher in the DN group than the ALS group, but this did not reach statistical significance. In the ALS group, Awaves were detected in at least 1 nerve in 13 patients in the DN group (92.85\%) versus 12 patients in the ALS group $(85.7 \%)(P=0.5483)$. Out of 64 nerves of patients in the $\mathrm{DN}$ group, A-waves were recorded from 25 nerves (39\%) and out of 61 nerves of patients in the ALS group and A-waves were recoded from 21 nerves $(34.4 \%)(P=0.6423)$.

In the DN group, A-waves were detected more often in nerves of upper extremities; out of a total of 25 nerves with A-waves, A-waves were recorded from 18 median and ulnar nerves $(72 \%)$ and the rest were recorded from peroneal and tibial nerves (28\%) and that was statistically significant $(P=0.0021)$. While in the ALS group, there was no significant difference in the occurrence of Awaves between upper and lower extremities; out of 21 nerves, A-waves were recorded from 11 nerves of the upper extremities $(52 \%)$ and 19 nerves of the lower extremities $(48 \%)(P=0.7979)$.

A-waves recorded from more than two nerves of the same patient were observed more frequently in patients with demyelinating neuropathies. Three patients out of 13 in the demyelinating neuropathy group $(23 \%)$ had more than two nerves with A-waves, compared to only one patient out of 12 in the ALS group (8.3\%). Yet, this did not reach statistical significance $(P=0.3257)$.

Complex A-waves (CAWs) occurred exclusively in patients with demyelinating neuropathy (DN) (Fig. 1). In patients with DN, out of 25 nerves with A-waves, complex A-waves were recorded from seven nerves $(28 \%)$ while the rest showed simple A-waves (Fig. 2). Most of the complex A-waves were recorded from the ulnar nerve (3), followed by the median (2) and then peroneal and tibial nerves (1 each). No CAWs were observed in ALS patients.

In both groups, A-waves were detected mostly in the absence of F-waves (72\% in DN group and $61.9 \%$ in ALS group, $P=0.4716$ ), followed by early A-waves (A-waves recorded before $\mathrm{F}$-waves) (24\% in DN group and $33.3 \%$ in ALS group, $P=0.4900$ ) and lastly, A-waves intermixed with F-waves (4\% in DN group and $4.76 \%$ in ALS group, $P=0.9009$ ). In both groups, we did not detect late Awaves (A-waves that occur after F-waves). Differences between both groups were statistically insignificant.

\section{Discussion}

A-waves were thought to be of clinically insignificant value; however, recently, they started to gain clinical importance and they are considered to be signs of peripheral nerve dysfunction [6,7]. In the current study, we aimed at characterizing A-waves in ALS and demyelinating neuropathies (DN). Two groups of patients were included, ALS group and DN group (14 patients each), and their nerve conduction studies were reanalyzed to look for A-waves. A-waves were assessed in 61 nerves in the ALS group versus 64 nerves in the DN group.

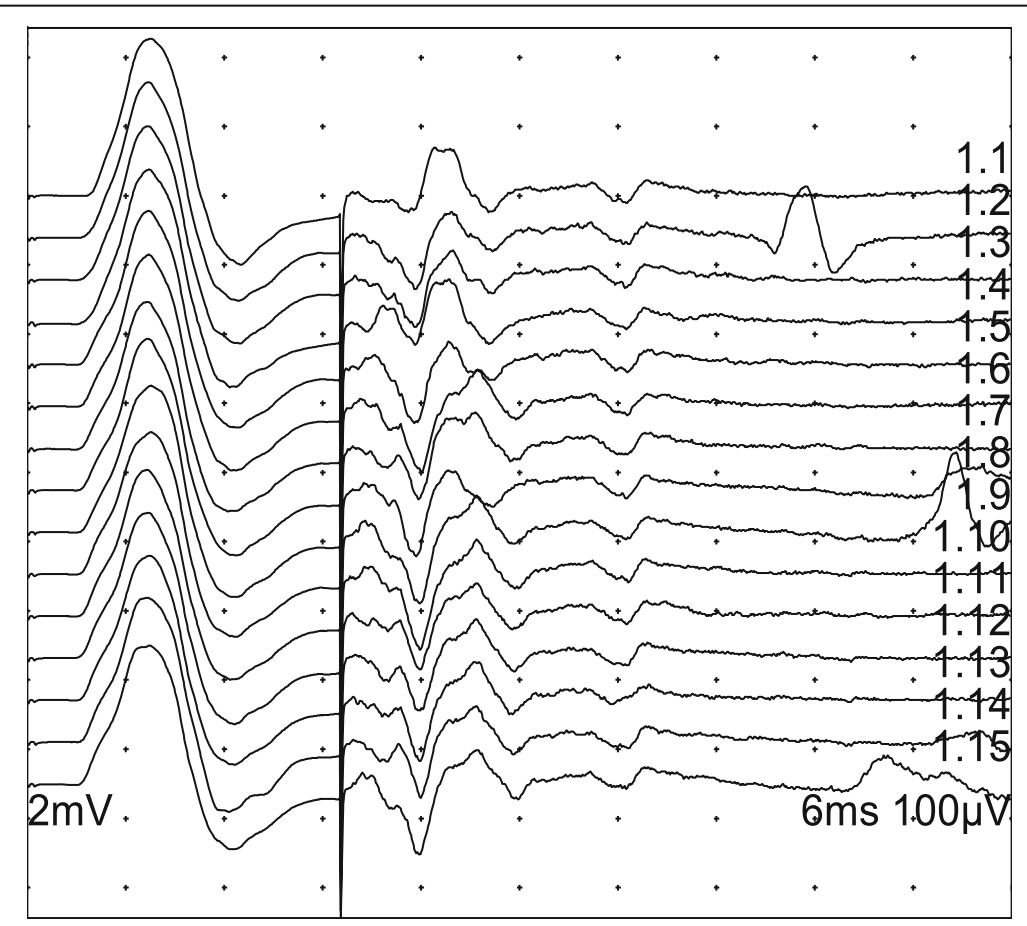

Fig. 1 CAWs in the right ulnar nerve 


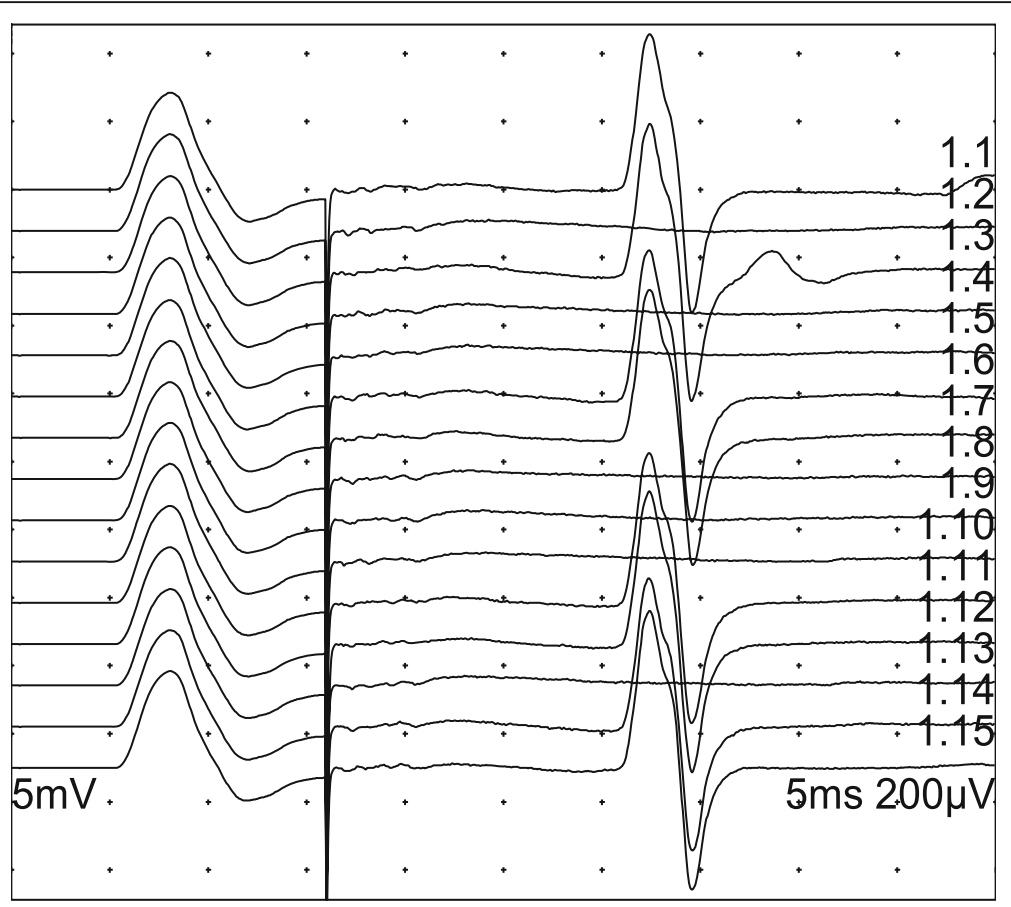

Fig. 2 Simple A-waves in the right median nerve

The frequency of occurrence of A-waves was higher in the DN group than the ALS group which agrees with other studies [7]. However, that did not reach statistical significance. The frequency of A-waves in the ALS patients in our study is higher than that reported in previous studies $[1,4]$. Also, the frequency of A-waves in patients with DN is slightly higher than other studies [9], this could be attributed to the small sample size.

In the current study, A-waves were recorded more frequently from the DN group, and they tend to occur in multiple nerves of the same patients, which is in accordance with other studies [8]. A-waves in the DN group were recorded more frequently from nerves of the upper extremities (median and ulnar). However, in the ALS group, there was no significant difference between nerves in the upper and lower extremities. One possible explanation for the presence of A-waves mostly in the median and ulnar nerves in the DN group is the predilection of some forms of DN (MMN) to affect upper limbs; median, ulnar, and radial nerves are most commonly affected in MMN [10].

In the current study, all A-waves detected in the ALS group were simple A-waves. However, complex A-waves (CAW) represented $28 \%$ of A-waves recorded from the DN group, and they were recorded more frequently from ulnar and median nerves. This was in accordance with other studies which found CAW a useful sign to distinguish between demyelinating neuropathies and anterior horn cell disorders $[7,8]$. Thus, and according to the abovementioned studies and the current study, CAW seems to be a useful specific electrodiagnostic sign to distinguish DN from ALS.

In the current study, we did not find a significant difference between both groups regarding the timing of occurrence of A-waves in relation to $\mathrm{F}$-waves. In both groups, Awaves with absent $\mathrm{F}$-waves were recorded more frequently, followed by early A-waves (recorded before F-waves) and then intermixed A-waves. Thus, this was not useful in differentiating between both conditions. The frequency of occurrence of early A-waves in the ALS group in the current study (33.3\%) was almost similar to that found in previous studies [4]. However, we found that the occurrence of Awaves in the absence of $\mathrm{F}$-waves was higher.

To the best of our knowledge, there were no previous studies comparing the characteristics of A-waves among ALS and DN patients, which gives credit to our work and will open the door to more studies in this area. Also, we included only patients that were newly diagnosed with ALS/DN and were not on specific treatment to make sure that our findings are not affected by medications, which again gives credit to our results. However, the study has some limitations that may have affected the results. The small sample size is one of the major limitations. Thus, we recommend a larger sample size for future studies to validate our results. Also, our sample was heterogeneous as patients within the same group were classified as definite, probable, or possible ALS/DN, which also could have contributed to the statistically insignificant results. Thus, a homogeneous sample is recommended in future studies. 


\section{Conclusion}

On some occasions, differentiating between ALS and DN is challenging, and since differentiation between both conditions is important for the prognosis and therapy, we need to find new tools for diagnosis. A-waves are recorded in anterior horn cell disorders and demyelinating neuropathies; however, characteristics differ between different conditions. A-waves in the DN group were recorded mainly from nerves of the upper extremities. CAWs occurred exclusively in DN. The presence of CAWs is supportive of demyelination, but their absence does not rule out the diagnosis. Future studies with larger and homogenous sample are needed to validate our results.

\section{Acknowledgements}

Not applicable

We confirm that we have read the Journal's position on issues involved in ethical publication and affirm that this report is consistent with those guidelines.

\section{Authors' contributions}

HRR analyzed the data and wrote the manuscript. The author read and approved the final manuscript.

\section{Funding}

We did not receive funds for this project.

\section{Availability of data and materials}

The manuscript does not contain any individual person's data in any form. The datasets generated during and/or analyzed during the current study are available from the corresponding author on reasonable request.

\section{Ethics approval and consent to participate}

This manuscript was approved by the local Ethical Committee of Neurology Department, Ain Shams University on 11 June 2019.

All patients signed consents to use their tests for research purposes.

\section{Consent for publication}

Not applicable.

\section{Competing interests}

The author declares that he/she has no competing interests.

Received: 27 July 2019 Accepted: 8 January 2020

Published online: 14 January 2020

\section{References}

1. Bischoff C, Stalberg E, Falck B, Puksa L. Significance of A-waves recorded in routine motor nerve conduction studies. Electroencephalogr Clin Neurophysiol. 1996;101:528-33. https:/doi.org/10.1016/S0013-4694(96)96553-4.

2. Brooks BR, Miller RG, Swash M, Munsat TL. El Escorial revisited revised criteria for the diagnosis of amyotrophic lateral sclerosis. Amyotroph Lateral Scler Other Motor Neuron Disord. 2000;1:293-9.

3. EFNS Task Force/ CME Article. European Federation of Neurological Societies/Peripheral Nerve Society Guideline on management of chronic inflammatory demyelinating polyradiculoneuropathy: report of a joint task force of the European Federation of Neurological Societies and the Peripheral Nerve Society — first revision. Eur J Neurol. 2010;17:356-63.

4. Fang J, Cui L, Liu M, Guan Y, Ding Q, Shen D, Li D, Tai H. A retrospective study of the characteristics and clinical significance of A-waves in amyotrophic lateral sclerosis. Front Neurol. 2017. https://doi.org/10.3389/fneur.00515.

5. Kornhuber ME, Bischoff C, Mentrup H, Conrad B. Multiple A waves in Guillain- Barre' syndrome. Muscle Nerve. 1999;22:394-9.

6. Lakshminarasimhan S, Venkatraman C, Vellaichamy K, Ranganathan L. Clinical significance of A waves in acute inflammatory demyelinating polyradiculoneuropathy. J Clin Neurophysiol. 2018;35(5):415-8.
7. Remiche G, Mavroudakis N. Supramaximally stimulated complex A-waves are helpful to distinguish lower motor neurone disease from demyelinating neuropathies. Neuromuscul Disord. 2014;24(9):911.

8. Rowin J, Meriggioli M. Electrodiagnostic significance of supramaximally stimulated A-waves. Muscle Nerve. 2000;23(7):1117-20.

9. Sartucci F, Bocci T, Borghetti D, Orlandi G, Manfredonia F, Murri L, Giannini F, Rossi A. Further insight on A-wave in acute and chronic demyelinating neuropathies. Neurol Sci. 2010;31(5):609-16.

10. Slee M, Selvan A, Donaghy M. Multifocal motor neuropathy: the diagnostic spectrum and response to treatment. Neurology. 2007;69(17):1680-7.

\section{Publisher's Note}

Springer Nature remains neutral with regard to jurisdictional claims in published maps and institutional affiliations.

\section{Submit your manuscript to a SpringerOpen ${ }^{\circ}$ journal and benefit from:}

- Convenient online submission

- Rigorous peer review

- Open access: articles freely available online

High visibility within the field

- Retaining the copyright to your article

Submit your next manuscript at $\boldsymbol{\nabla}$ springeropen.com 